\title{
TMJ ARTHROSCOPY: "CAPSULAR MIGRATION" IN INTERNAL DERANGEMENT; IS ARTHROCENTESIS A RELIABLE SUBSTITUTE TO ARTHROSCOPY?
}

\author{
Waleed R. El- Beialy,*** and Nadia Galal*
}

\begin{abstract}
Internal derangement (ID) of the TemporoMandibular Joint (TMJ) is a general orthopedic term implying a mechanical fault that interferes with the smooth action of a joint. Athroscopy and arthrocentesis of superior TMJ compartment are among the commonly used diagnostic and minimally invasive treatment modalities for ID. Arthrocentesis has been considered as the cheaper, simpler and blind substitute for arthroscopy. This study reports arthroscopic outcomes that defy this understanding, and presents a new arthroscopic finding in ID patients: "Capsular Migration"; which changes the criteria of a successful entry into the superior TMJ compartment.
\end{abstract}

KEYWORDS: Internal Derangement, TMJ Arthroscopy, Arthrocentesis, TMJ disc displacement, Surgical Arthroscopy

\section{INTRODUCTION}

The TemporoMandibular Joint (TMJ) is the unique structure that facilitates the complex articulation of the mandible to the base of the skull, through its upper bony component the glenoid fossa and eminence. Internal derangement (ID) of the TMJ was defined as an abnormal relation between the articular disc with respect to the mandibular condyle, the temporal fossa and the temporal eminence. ${ }^{(1)}$ Recently, ID of a synovial joint is not considered as a disease. The biomechanical joint dysfunction that is associated with ID represents a failure of the intra-articular tissues caused by the loss of the structure and function. (2) Being of an unclear and multifactorial etiology remains as one of the few generally accepted statements about ID. ${ }^{(3,4)}$ The classifications of ID numerous since Dolwick et al classification in 1983, (1) through Katzberg (1989), (5) Wilkes staging (1989), (6) Drace and Enzmann (1990), ${ }^{(7)}$ and the latest wider perspective Research Diagnostic Criteria for TMDs RDC/ TMD ${ }^{(8)}$ (more recently updated to the Diagnostic Criteria [DC] for TMDs “DC for TMD”). ${ }^{(9,10)}$ There is no contradiction between various classifications, but rather attempts to establish a clear outline to pave the way for the most ideal treatment and hence

* Associate Professor of Oral \& Maxillofacial Surgery Department, Faculty of Dentistry, Cairo University

** Associate Professor of Oral \& Maxillofacial Surgery Department, Faculty of Dentistry, Future University in Egypt (FUE) 
best prognosis. The current arthroscopic study included Wilkes stages II (Early/Intermediate) to IV (Intermediate/Late), ${ }^{(6)}$ or Axis I TMD according to DC for $\mathrm{TMD}^{(9)}$ or Joint disorders/ Disc disease (2-A) according to AAOP Taxonomic classification. ${ }^{(10)}$ In Israel's ${ }^{(2)}$ latest remarkable review about the history of ID treatment, it was clearly highlighted there were changing perspectives on management; from appliance and surgical disc repositioning back in the 1970s, to arthroplasties with/out discoplasty and discectomy with/out replacements up until the mid 1990s. A change in the approach to treat ID was lead by various MRI studies demonstrating the high percentage of disc displacement in asymptomatic patients (32-38\%); raising the question about the value of repositioning the disc. (2) Arthroscopy was considered a breakthrough in the management of ID, providing a conservative resort to eliminate symptoms, alleviate pain and improve function. ${ }^{(11,12,13,14)}$ Arthrocentesis was laterintroduced as an even simpler approach requiring no setups and was claimed to be safe and effective. ${ }^{(15,16,17)}$ Meta-analysis concluded that the most significantly successful results in surgical treatment of disc displacement without reduction were arthroscopic surgery and arthrocentesis. ${ }^{18)}$ Similarly, a more recent meta-analysis and review of literature suggested that arthroscopy yielded superior efficacy to arthrocentesis in increasing joint movement and decreasing pain in the management of ID. ${ }^{(14)}$

The points of needle/trocar insertion had been subject to several modifications ever since their debut by Ohnishi ${ }^{(19)}$ for arthroscopic lysis and lavage. Murakami et al. (20) first described a technique of TMJ arthrocentesis with pumping irrigation and hydraulic pressure to the upper joint cavity followed by manipulation of the jaw. Nitzan two-needle arthrocentesis (15) was performed by the method described later on by McCain et al (21) for arthroscopy where a canthotragal line is drawn form lateral canthus to the most posterior and central point on the tragus (Holmlund-Hellsing Line) ${ }^{(22)}$ along which the posterior point is located $10 \mathrm{~mm}$ from middle of the tragus and $2 \mathrm{~mm}$ below the canthotragal line, and the anterior point of entry is placed $10 \mathrm{~mm}$ anterior to the previous point and $10 \mathrm{~mm}$ below it. ${ }^{(21)}$ Laskin et al ${ }^{(23)}$ suggested the insertion of the anterior needle only $2-3 \mathrm{~mm}$ anterior to the first entry for arthrocentesis as access into the anterior recess is not necessary as during arthroscopic visualization of the superior TMJ compartment. Still regarding arthroscopy, a modification was suggested where second entry point was even posterior $(3 \mathrm{~mm})$ to the first, at $7 \mathrm{~mm}$ anterior from the middle of the tragus and $2 \mathrm{~mm}$ inferior along the canthotragal line. ${ }^{(24)}$ While others reported using single entry point at the same site $10 \mathrm{~mm}$ anterior to tragus and $2 \mathrm{~mm}$ inferior to canthotragal line. ${ }^{(25,26)}$ Apart from the location and number of points of insertion of trocar/needle, arthroscopic approaches have been categorized into: superior posterolateral approach (most common), inferior posterolateral approach, superior anterolateral approach, inferior anterolateral approach and the endaural approach. Literature has also pointed out that $100-300 \mathrm{ml}$ of fluid were sufficient for mobilization of the disc, washout and neutralization of degraded particles and inflammatory components, and intra-articular pressure reduction. Arthrocentesis has been reported to reduce joint pain, improve function, and reduce clicking. ${ }^{(27)}$ On the other hand, TMJ arthroscopy has been used to treat patients with painful clicking or popping, to release intra-articular adhesions and anteriorly displaced non-reducing discs, and to confirm other diagnostic findings that could warrant surgical intervention. ${ }^{(23)}$

\section{PATIENTS AND METHODS}

In this retrospective clinical study, $76 \mathrm{TMJ}$ arthroscopies were carried out as a one-day surgery, under general anesthesia (18 unilateral TMJ patients $14 \%$; and 29 bilateral TMJ patients $86 \%$ ) via routine oral \& maxillofacial naso-tracheal intubation. A 
sterile cotton pellet was placed in the external auditory canal followed by routine surgical field scrubbing with povidone iodine solution and the patient was draped in the usual manner with sterile adhesive sheets. Patients suffering varying degrees of ID: namely disc displacement with reduction, and disc displacement without reduction (Wilkes classes II-IV). All chronic cases were conservatively treated using pharmacotherapy and splint therapy for a minimum of 3 months as highlighted in our previous publication; ${ }^{(4,28)}$ and only those who failed to improve underwent TMJ arthroscopy. Acute closed lock patients underwent immediate arthroscopic lysis and lavage. Demographic data presented in (Figure 1).

Distension of the superior compartment was performed in order to avoid iatrogenic damage to the cartilaginous surfaces. Upon introduction of the trocar/cannula in the depression left behind the anteriorly pulled condyle by the assistant surgeon who distracted the mandible anteriorly and inferiorly; a 21-gauge needle-mounted syringe filled with 2-3 ml Ringer's lactated solution, was then introduced through the puncture point into the superior joint space for further insufflation and distention. Normal resistance was felt after injection of approximately 2-3 $\mathrm{ml}$ indicating that the joint space was filled; the increased intra-articular pressure caused a slight downward movement of the jaw and confirmed the correct deposition of the solution. Arthroscopic trocar/cannula insertion points were mainly performed anatomically, where the condylar head was palpated in both open and closed positions, and drawn on the skin. The Holmlund-Hellsing Line was also drawn and the two entries points were initially those reported by McCain. (21) Triangulation was achieved to allow for visualization of the working ends of surgical hand instruments or motorized shavers or ablation
A

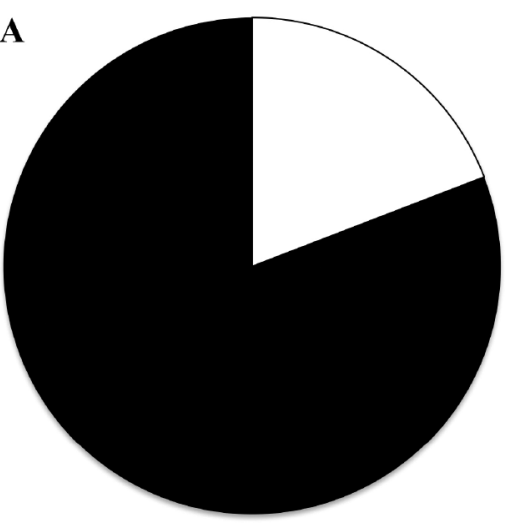

$\square$ Male

- Female
B

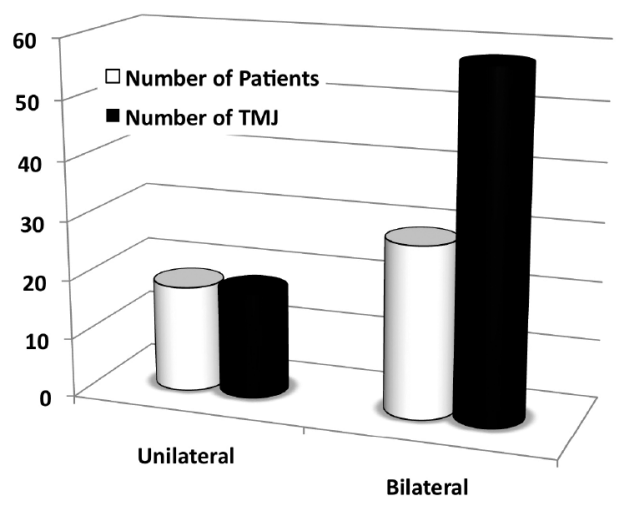

Fig. (1) Demographic data of the study. (A) Male to female ratio $(9: 38)$; (B) Unilateral to bilateral ID cases (18:58 TMJ; and 18:29 patients); (C) Disc displacement with reduction acute to chronic cases ratio (8:39) and Disc displacement without reduction acute to chronic cases ratio $(9: 20)$. 
tips introduced through the second working cannula (Figure 2) The arthroscopic setup included $2.4 \mathrm{~mm}$ arthroscopes ( $30^{\circ}$ and $70^{\circ}$ angle lenses) with $2.7 \mathrm{~mm}$ outer cannula (single and double stop-cocks) (Karl Storz)

All surgeries were initiated for hydraulic lysis and lavage of the TMJ superior compartment. Surgical instruments were only utilized following failure of release of adhesions hydraulically. Ablation was minimally used to cases with intraarticular bleeding that was uncontrolled by neither increasing intra-articular pressure nor compressions following shaver lysis of adhesions. All surgeries included washes by a minimum of $1 \mathrm{~L}$ of saline or Ringer's lactated solution. The second surgical assistant carried out maneuvering the TMJ along its full range of motion, at various intervals during the surgery. Fluid pumps as well as, high volume suction were used. Upon completion of lysis, lavage and surgical procedure, the superior TMJ compartment was evacuated from all fluids by syringe aspiration or using the high volume suction, and $2 \mathrm{ml}$ of betamethasone were introduced into the
TMJ following removal of one cannula. The TMJ was maneuvered in the full range of motion (ROM) again following removal of all cannulas.

Immediately following recovery, self-employed exercises of the TMJ was carried out by the patients and were instructed to perform such training on evenly divided daily sessions for the first 2 weeks postoperatively. Normal diet was strictly advised to make use of the surgical outcome achieved and to avoid the recurrence of adhesions. All patients had custom thick soft splints delivered immediately following surgery, and those with residual clicking were appointed anterior repositioning splints for at least the $1^{\text {st }}$ postoperative month. NSAIDs and central acting muscle relaxants were prescribed and tapered over the first 2 weeks postoperatively. Visual analogue scales (VAS) were carried out for pain, headaches, pre-auricular tenderness, and chewing ability prior and postsurgical during follow-up. The maximum interincisal opening (MIO), and ROM were also documented. The patients were followed up for a minimum of 6 months postoperatively, and biannually afterwards.

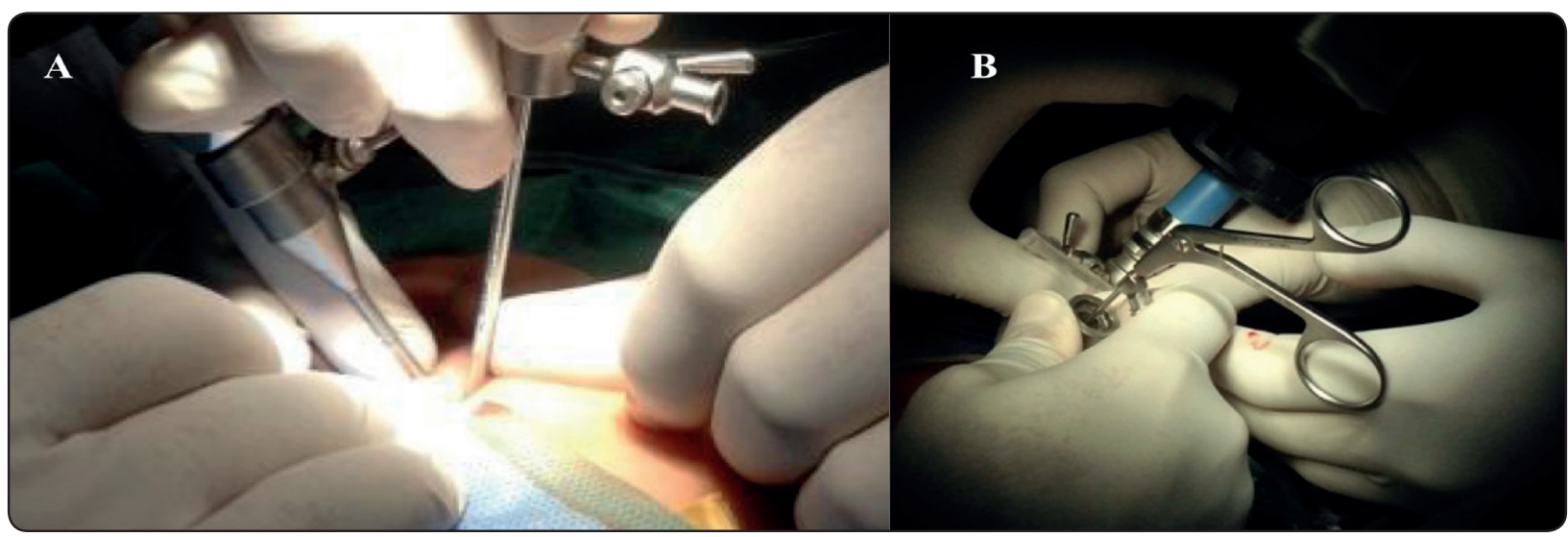

Fig. (2) Double puncture arthroscopy. (A) Triangulation of the 2 cannulas for visualization of the working ends. (B) Hand instrument (Grasper forceps-Crocodile) in one cannula, for hand-driven removal of adhesions and fibrosis and the arthroscopic lens mounted to camera in the second cannula.

* Karl Storz, Tuttlingen, Germany 


\section{RESULTS}

Most patients had drastic improvement in their symptoms immediate postoperatively, except for 2 cases, who had persistent tenderness pre-auricular for almost 1 and 1.5 months postoperatively. The MIO was improved to an average of $38.5 \mathrm{~mm}$ from an initial average of $18 \mathrm{~mm}$ preoperatively, and the improvement was maintained over 6 months of follow-up. The VAS for pain, headaches, preauricular tenderness, and chewing ability were generally improved throughout the 6 months postoperative follow-up.

The initial 100-300ml of fluid used for lysis and lavage were insufficient for release all adhesions within the superior TMJ compartment. TMJ arthroscopy was carried out using 1-3L of fluid, and more fluid was needed when the fluid pump and high volume suction were used. Intermittent opening and closure of the outflow line (or suction) along with the inflow was repeatedly performed in order to buildup reasonable pressure within the TMJ to release the adhesions and blood clots.

Upon the insertion of the first trocar at the designated point in a case with extremely limited mouth opening preoperatively $(13 \mathrm{~mm})$, the arthroscope revealed that we were retrocapsular (extracapsular). On an attempt to insert the second trocar/cannula in the $2^{\text {nd }}$ point $(20 \mathrm{~mm}$ from the tragus anteriorly, and $10 \mathrm{~mm}$ inferior to the canthotragal line), it was still retrocapsular; and the only way to enter the capsule was to strictly followup what was thought to be the bizarre anatomy of the case, despite being too far anteriorly (around $35 \mathrm{~mm}$ ). The arthroscopic findings were compared to the MRI, of the patient. The patient (female) was suffering from anterior disc displacement without reduction, in which the articular disc was too far anteriorly (Figure 3). Strangely, this was the case in another $8 \mathrm{TMJ}$ (total of 9), where the disc was displaced too far anteriorly. However, some were still experiencing late clicks (anterior disc displacement with reduction). There were blood clots seen retrocapsular as well as, what seemed like fibrosis in all these patients. Strangely enough in those cases, the initial insufflations of what was thought to be the superior TMJ compartment prior to arthroscopic insertion, led to the movement of the mandibular anterior teeth, as if it was actually within the superior TMJ compartment. Moreover, there was a continuous flow of fluid from the inflow and through the outflow -both of which were retrocapsular- as seen in routine successful arthrocentesis. Strangely enough, lysis and lavage of retrocapsular (extracapsular) tissues led also to improvement in the patients' initial signs and symptoms. (Figure 3)

\section{DISCUSSION}

Arthroscopy and arthrocentesis are well documented throughout the literature as less invasive and associated with minimal complications, they have recently replaced open operations for patients with dysfunction of the TMJ that failed to respond to conservative treatment. ${ }^{(27,29)}$ Clearly, arthroscopic lysis and lavage have more possible complications than arthrocentesis, which is clinic-based, costeffective, and minimally invasive. ${ }^{(27)}$ It is not the authors' intention to investigate the effectiveness of such procedures in the management of ID cases, as it is well documented throughout the literature ${ }^{(4,27,28)}$ However, arthrocentesis is a clinic-based technique to washout inflammatory exudates and mediators as bradykinin, interleukin-6, proteins, denatured hemoglobin and various proteinases. ${ }^{(30,31)}$ The reported fluids whether it be isotonic saline or Ringer or Ringer's lactated solution were usually along the volume of $100-300 \mathrm{ml}$. It should be noted that such amount was not sufficient to dislodge fibrotic tissues and adhesions arthroscopically, and that a minimum of $1 \mathrm{~L}$ (range 1-3L) are necessary for arthroscopic surgery. Of course, this volume is not applicable for arthrocentesis under local anesthesia and clinic settings; nor is it tolerable by a conscious patient. The use of hydraulic fluid pump reaching pressure 


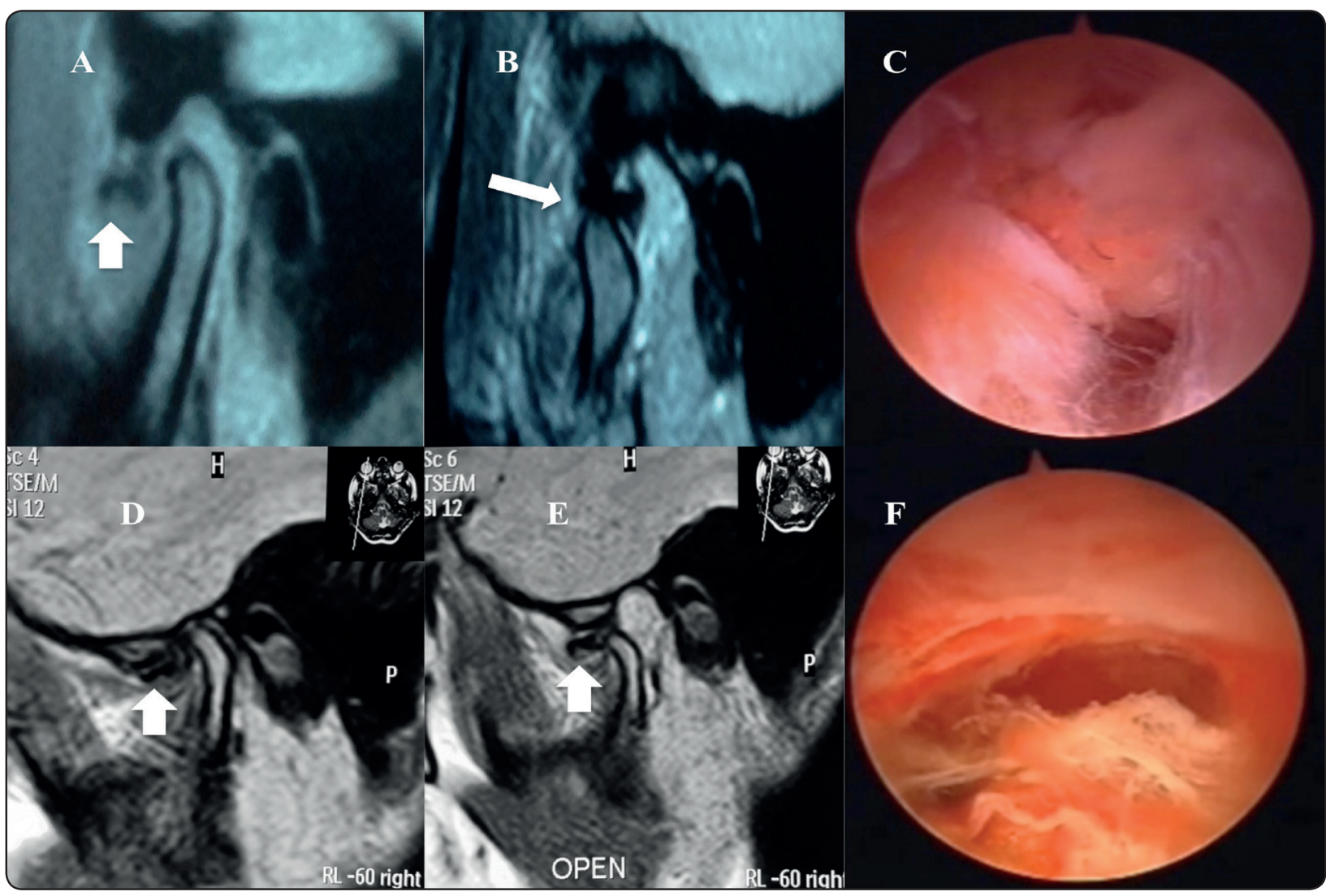

Fig. (3): Capsular Migration: (A) MRI of an Anterior Disc Displacement (closed mouth); (B) with reduction (open mouth); (D) MRI of an Anterior Disc Displacement (closed mouth); (E) without reduction (open mouth); (C) The extracapsular (retrocapsular) view of the TMJ capsule as seen through arthroscope; (F) A posterior view of the capsule along with ruptured posterior capsular fibers. (White arrow pointing to TMJ disc in MRI)

of around $240 \mathrm{mmHg}(\sim 40 \mathrm{kPa})$ had failed to release fibrosis and adhesions; and necessitated the use of surgical shaver and cutters. Which in a sense is in agreement with Yura and Totsuka whom advocated the superiority of high-pressure arthrocentesis compared to low pressure one. ${ }^{(32,33)}$ Yet, this study questions their conclusion, or rather underline the word "can" in their conclusion: "can remove adhesions and widen the joint space, the technique might be useful for patients with closed lock and adhesions." Some superior TMJ compartments were clearly pseudo-divided with such adhesions, preventing the flow of fluid from the posterior recess to the anterior one, and even within the posterior recess. This in turn raises doubts about the validity of single point arthrocentesis into the posterior recess, and whether it is actually in the TMJ superior compartment. ${ }^{(24-26)}$ Hence, distinction between the two techniques (arthroscopy and arthrocentesis) and their scope should be clearly highlighted. Arthrocentesis should not be regarded as a substitute for arthroscopy, but rather as a technique to flush inflammatory exudates, so it is rather a lavage. Moreover, this study questions the ability of arthrocentesis to release adhesions of fibrosis, especially with small gauge needles.

This study reports a phenomenon of "Capsular Migration"; where the whole TMJ capsule migrates anteriorly along with the anteriorly displaced disc. This phenomenon was noted in 9 out of 76 TMJ included in this study (12\%). While those patients had varying degrees of displacement, (with and without reduction), they seemed to share the following: too far anteriorly displaced disc and a long history of 
ID (chronic). Regarding the points of insertion of needle/trocar into the upper TMJ compartment, the statement of Tozoglu et al "The fossa portal puncture site should be confirmed by palpation. However, anatomical variations should be kept in mind." is critical for proper entry into the superior TMJ compartment based on the current study's results. While Holmlund-Hellsing Line (canthotragal line) and the suggested entry points ${ }^{(27)}$ are valid guidance points; yet, the priority is for palpation of the condyle. The hereby-proposed Capsular Migration phenomenon, raises doubts concerning the single entry posterior arthrocentesis points, ${ }^{(24,25,26)}$ and whether such fluid flow is within the TMJ capsule or retrocapsular; giving the outcome of this study. Moreover, the improvement of patients who received retrocapsular lysis and lavage, necessitates further studies, about our understanding of ID and its management.

Contrary to recommended postoperative nonchewing soft diet for a few days, ${ }^{(27,29)}$ this study reports better outcome with immediate postoperative normal chewing habits; yet, in agreement with the outcome of immediate exercises of range of movement. ${ }^{(27)}$ Similar to many previous researches ${ }^{(27)}$ this study reports arthroscopic lysis and lavage of the upper joint compartment through two entries provides good short and long-term results.

\section{CONCLUSIONS \& RECOMMENDATIONS}

- Arthocentesis is a lavage only clinic-based technique, with very limited capability of lysis.

- High-pressure lysis and lavage is more efficient in releasing adhesions.

- Athrocentesis is not a substitute for arthroscopy.

- Single point entry into the superior TMJ compartment is questionable regarding its ability to flush the full joint space.

- Capsular Migration phenomenon is proposed and anticipated in chronic cases of
ID, where the disc is displaced too far anteriorly.

- Anatomical palpation of the TMJ condyle as a guide for trocar/needle insertion is of higher priority than the reported points in literature, which are only guidelines.

\section{Limitations}

Further multicenter studies on larger number of patients with ID are necessary to confirm or exclude the proposed phenomenon "Capsular Migration".

\section{Ethical approval}

The work did not require approval by the appropriate ethical committees related to the institutions in which it was performed.

\section{REFERENCES}

1. Dolwick M, Riggs R. Diagnosis and treatment of internal derangements of the temporomandibular joint. Dent Clin North Am. 1983; 27: p. 561.

2. Israel HA. Internal Derangement of the Temporomandibular Joint New Perspectives on an Old Problem. Oral Maxillofacial Surg Clin N Am. 2016; 28: p. 313-333.

3. Dworkin S, Huggins K, LeResche L, Von Korff M. Epidemiology of signs and symptoms in temporomandibular disorders : clinical signs in cases and controls. J Am Dent Assoc. 1990; 120: p. 273-281.

4. ElYazby W, El Sharrawy E, Mekhemer S, El Sholkamy M, El-Beialy W. Correlation between clinical signs and symptoms and the arthroscopic findings in TMJ internal derangement (ID). Egyptian Dental Journal. 2016 October; 62: p. 4617-4623.

5. Katzberg R. Temporomandibular joint imaging. Radiology. 1989; 170: p. 297.

6. Wilkes C. Internal derangements of the temporomandibular joint. pathological variations. Arch Otolaryngol Head Neck Surg. 1989.; 115: p. :469.

7. Drace J, Enzmann D. Defining the normal temporomandibular joint: closed, partially open, and open mouth MR imaging of asymptomatic subjects. Radiology. 1990; 177: p. 67. 
8. Dworkin S, LeResche L. Research diagnostic criteria for temporomandibular disorders: review, criteria, examinations and specifications, critique. J Craniomandib Disord. 1992; 6: p. 301-55.

9. Schiffman E, Ohrbach R, Truelove E, Look J, Anderson $\mathrm{G}$, Goulet J, et al. Diagnostic criteria for temporomandibular disorders (DC/TMD) for clinical and research applications: recommendations of the international RDC/TMD consortium network and orofacial pain special interest group. J Oral Facial Pain Headache. 2014; 28(1): p. 6-27.

10. Peck C, Goulet J, Lobbezoo F, Schiffman E, Alstergren P, Anderson G, et al. Expanding the taxonomy of the diagnostic criteria for temporomandibular disorders. J Oral Rehabil. 2014; 41(1): p. 2-23.

11. Moses J, Poker I. TMJ arthroscopic surgery: an analysis of 237 patients. J Oral Maxillofac Surg. 1989; 47: p. 790.

12. McCain J, Sanders B, Koslin M, Quinn J, Peters P, Indresano A. Temporoman- dibular joint arthroscopy: A 6-year multicenter retrospective study of 4,831 joints. J Oral Maxillofac Surg. 1992; 50: p. 926.

13. Murakami K, Moriya Y, Goto K, Segami M. Four-year follow-up study of temporomandibular joint arthroscopic surgery for advanced internal derange- ments. J Oral Maxillofac Surg. 1996; 54: p. 285.

14. Al-Moraissi E. Arthroscopy versus arthrocentesis in the management of internal derangement of the temporomandibular joint: a systematic review and meta-analysis. Int J Oral Maxillofac Surg. 2015; 44(4): p. 104-12.

15. Nitzan D, Dolwick M, Martinez G. Temporomandibular joint arthrocentesis: a simplified treatment for severe limited mouth opening. J Oral Maxillofac Surg. 1991; 49(1): p. 1163-7.

16. Dimitroulis G, Dolwick M, Garza AM A. Temporomandibular joint arthrocentesis and lavage for the treatment of closed lock: a follow-up study. Br J Oral Maxillofac Surg. 1995; 33: p. 23-7.

17. Hosaka H, Murakami K, Goto K, Iizuka T. Outcome of arthrocentesis for temporomandibular joints with closed lock at 3 years follow-up. Oral Surg Oral Med Oral Pathol Oral Radiol Endod. 1996; 82: p. 501-4.

18. Reston J, Turkelson C. Meta-analysis of surgical treatments for temporomandibular articular disorders. J Oral Maxillofac Surg. 2003; 61: p. 3-10.

19. Ohnishi M. Arthroscopy and arthroscopic surgery of the temporomandibular joint (TMJ). Rev Stomatol Chir Maxillofac. 1990; 91: p. 143-50.

20. Murakami K, Iizuka T, Matsuki M, Ono T. Recapturing the persistent anteriorly displaced disk by mandibular manipu- lation after pumping and hydraulic pressure to the upper joint cavity of the temporomandibular joint. Cranio. 1987; 5: p. 17-24.

21. McCain J, de la Rua H, LeBlanc W. Puncture technique and portals of entry for diagnostic and operative arthroscopy of the temporomandibular joint. Arthroscopy. 1991; 7: p. 221-32.

22. Holmlund A, Hellsing G. Arthroscopy of the temporomandibular joint: an autopsy study. Int J Oral Surg. 1985; 14: p. 169-75.

23. Laskin D, Greene C, Hylander W. Temporamandibular disorders: an evidence-based approach to diagnosis and treatment. Laskin DM GCHW, editor. Chicago: Chicago Quintessence; 2006.

24. Alkan A, Etöz O. A new anatomical landmark to simplify temporomandibular joint arthrocentesis. Br J Oral Maxillofac Surg. 2010; 48: p. 310-311.

25. Alkan A, Bas B. The use of double-needle cannula method for temporomandibular joint arthrocentesis: clinical report. Eur J Dent. 2007; 1: p. 179-82.

26. Rehman K, Hall T. Single needle arthrocentesis. Br J Oral Maxillofac Surg. 2009 July; 47: p. 403-4.

27. Tozoglu S, Al-Belasy FA, Dolwick M. A review of techniques of lysis and lavage of the TMJ. British Journal of Oral and Maxillofacial Surgery. 2011; 49: p. 302-309.

28. ElYazby W, El Sharrawy E, Mekhemer S, El Sholkamy M, El-Beialy WR. Temporomandibuar joint chronic closed lock: Spontaneous resolution folowing surgical arthroscopy. Future Dental Journal. 2016; 6: p. 80-85.

29. Nitzan D. Arthrocentesis-incentives for using this minimally invasive approach for temporomandibular disorders. Oral Maxillofac Surg Clin North Am. 2006; 18: p. 311-28.

30. Zardeneta G, Milam S, Schmitz J. Elution of proteins by contin- uous temporomandibular joint arthrocentesis. J Oral Maxillofac Surg. 1997; 55: p. 709-17.

31. Kaneyama K, Segami N, Nishimura M, Sato J, Fujimura. The ideal lavage volume for removing bradykinin, interleukin-6, and protein from the temporomandibular joint by arthrocentesis. J Oral Maxillofac Surg. 2004; 62: p. 657-61.

32. Yura S, Totsuka Y, Yoshikawa T, Inoue N. Can arthrocentesis release intracapsular adhesions? Arthroscopic findings before and after irri- gation under sufficient hydraulic pressure. J Oral Maxillofac Surg. 2003; 61: p. 1253-6.

33. Yura S, Totsuka Y. Relationship between effectiveness of arthrocentesis under sufficient pressure and conditions of the temporomandibular joint. J Oral Maxillofac Surg. 2005; 63: p. 225-8. 